ITC 3/47

Journal of Information Technology and Control

Vol. 47 / No. 3 / 2018

pp. 564-574

DOI 10.5755/j01.itc.47.3.18451

(C) Kaunas University of Technology
Robust Quantized Hœ ILC Design for Uncertain Systems with Communication Constraints

Received 2017/06/23
Accepted after revision 2018/07/30

\title{
Robust Quantized Ho ILC Design for Uncertain Systems with Communication Constraints
}

\section{Bu Xuhui}

School of Electrical Engineering \& Automation, Henan Polytechnic University, Jiaozuo, China. Institute of Artificial Intelligence \& Control, Qingdao University of Science and Technology, Qingdao, China e-mail:buxuhui@gmail.com

\section{Cui Zhongyuan}

College of Computer Science and Technology, Zhoukou Normal University, Zhoukou, China, e-mail: cuizy@zknu.edu.cn;

\section{Cui Lizhi, Qian Wei}

School of Electrical Engineering \& Automation, Henan Polytechnic University, Jiaozuo, China, e-mail: clzh0308@hpu.edu.cn; qwei@hpu.edu.cn

Corresponding author: buxuhui@gmail.com

In this paper, an Ho ILC algorithm is designed for network-based uncertain systems with communication constraints, where the system is suffering from data dropouts and data quantization. Here, it is assumed that the system state and tracking error are first quantized through a logarithmic quantizer, which can decode these data into finite quantization levels. Furthermore, the model of packet dropout is described as the Bernoulli binary value sequences with known probability. Then, the 2-D dynamic of such ILC process is established by a stochastic Roesser model. To deal with the quantization error, the sector bound method is also utilized. A sufficient condition is obtained for such systems and the condition can realize the design of the ILC controller. The effectiveness of the proposed design is validated by application to an injection molding process.

KEYWORDS: Iterative learning control, communication constraints, 2-D Roesser system, Robust Ho design.

\section{Introduction}

Iterative learning control was first proposed by Airimoto in 1984 [4]. It is an effective tracking con- trol approach for dynamic systems that performs repeated tasks over a finite time interval. After three 
decades of developments, ILC has made considerable theoretical achievements. Meanwhile, ILC is widely used in many practical systems, for instance, robotic systems, traffic control systems, industrial batch processes and hard disk drives $[1,4,6,12,13,22,23$, $29,31,33]$. Most of the existing ILC literature is considered for the conventional point-to-point control systems, where the information can fully transmit between the controller and the plant. In practice, networked control systems (NCSs) have aroused widespread concern and application $[3,16,19,20,27,35$, 36]. Compared with point-to-point control systems, NCSs have some advantages such as easy installation, high security and low maintenance cost. However, due to the limitation of network bandwidth and the influence of transmission channel, it is easy to cause some incomplete information, such as data dropout, communication delay and data quantization.

Some research results have been obtained for the iterative learning control systems with data losses. In [2], a packet dropout compensation approach is proposed by using ILC for networked control systems. It is shown that this design can relax the result of critical data dropout rate, which will change the stability of NCSs. In [26], an averaging ILC scheme is introduced to nonlinear systems with packet dropouts, where the one step network-induced delay can also be seen as packet dropout. It is shown that the missing data can be compensated by the average of previous data. In [8], the convergence of nonlinear ILC systems with packet losses is discussed, where the situation considered is one in which data dropout occurs simultaneously both on the control input side and on the measurement output side. The main results of [8] are that the ILC system still guarantees convergence even though there exists data packet loss. In [9], by using super-vector formulation, an Hळ ILC design method is developed where the $\mathrm{H} \infty$ performance is defined along iteration axis and the ILC controller design is discussed under the framework of stochastic systems. In [11], a 2-D stochastic Roesser model is established for discrete time linear ILC systems with random data dropouts. Based on this formulation, the problem of learning gain design can be transformed into the mean square stability for such established 2-D system. In [14], a stochastic approximation ILC algorithm is developed for a class of nonlinear systems with measurement missing, where the control direction of the system is also unknown.
On the other hand, data quantization is also an important issue for NCSs [7, 17, 25, 30]. That is because the data need to be quantized before being transferred to the next network node owing to the limited transmission capacity of the network. In our previous work [10], the data quantization is first discussed for ILC systems, where the data quantization occurs at the measurement output side. Some convergence conditions are established for both linear systems and nonlinear systems. The main result shows that, due to the effect of data quantization, the tracking error converges to a bound relying on the quantization level. Large quantization density will cause large tracking error. Hence, it is an interesting work to design an ILC algorithm to reduce the influence of data quantization.

This paper focuses on the problem of ILC design for network-based uncertain systems with packet dropouts and data quantization. The objective is to design an ILC algorithm so that the impact of data quantization and missing can be reduced. It is noticed that the 2-D Roesser model is an effective tool to describe the dynamical behavior of ILC systems [21, 24, 32]. Hence, here we also first establish the 2-D Roesser model for such ILC processes to design the controller. To describe the communication constraints, Bernoulli processes are introduced for modeling packet dropouts and the logarithmic type quantizer is selected to compress the system data. The stochastic mean-square asymptotical stability is also introduced in this paper to establish the stability of such 2-D system. A sufficient asymptotical stability condition is given for the 2-D system with an $\mathrm{H} \infty$ disturbance attenuation level by using the sector bound method, and the stability condition can realize the design of the ILC controller.

There are at least two differences between this paper and existing works. Firstly, comparing with the existing ILC design based on 2-D model in [28], this paper considers the effect of communication constraints and iteration-varying disturbances. The stability analysis is given under the framework of stochastic systems, and the proposed controller has an $H_{\infty}$ disturbance attenuation level. Secondly, comparing with the result in [10], the proposed design can reduce the influence of the data quantization. The algorithm proposed in this paper can get zero tracking error if the disturbance tends to zero, thus [10] can only obtain bounded tracking error. The main contribution of this paper can be summarized as follows: (1) 
The ILC design is considered for linear systems with communication constraints, where the data dropout and data quantization occur due to the limitation of transmission channel. (2) A 2-D stochastic Roesser model is established to describe the dynamics of the ILC system and then the problem of ILC design can be transformed into the synthetic of the 2-D system.

The rest of the paper is organized as follows. Section 2 introduces the NCSs framework for the ILC systems. Section 3 gives a sufficient asymptotical stability condition for the established 2-D system. The condition can realize the design of the ILC controller. Section 4 supplies a numerical example to validate the effectiveness of the proposed design.

Notation: Let $T$ denote the matrix transposition and $R^{n}$ denote the $n$-dimensional Euclidean space and $I$ and $O$ stand for identity matrix and zero matrix, respectively. diag $\{\cdot\}$ stands for the standard diagonal matrix. A matrix without special instructions has an appropriate demission to ensure that the algebraic operations are compatible. $E\{x\}$ denotes the expectation of $x$ and $E\{x \mid y\}$ stands for the condition expectation.

\section{Problem Formulation}

Let us consider the following discrete time linear repetitive system

$$
\left\{\begin{array}{rl}
x(t+1, k)= & (A+\Delta A) x(t, k)+(B+\Delta B) u(t, k) \\
& +B_{1} w(t, k) \\
y(t, k)=C x(t, k)
\end{array},\right.
$$

where the subscript $k$ denotes iteration and $t$ denotes discrete time. $x(t, k) \in R^{n}, y(t, k) \in R^{l}$, $u(t, k) \in R^{m}, w(t, k) \in R$ are state, output and input variables and iteration-varying disturbances. $A, B, C, B_{1}$ are the system matrices with appropriate dimension. $\Delta A$ and $\Delta B$ are uncertain perturbations of $A$ and $B$. They can be described as

$$
\begin{aligned}
& \Delta A=E \Sigma F_{1}, \\
& \Delta B=E \Sigma F_{2},
\end{aligned}
$$

where $\Sigma$ is an uncertain matrix satisfying $\Sigma^{T} \Sigma \leq I$. $E, F_{1}, F_{2}$ are constant matrices used to describe the determinate parts of the uncertain perturbations, which can also reflect the structures of $\Delta A$ and $\Delta B$. $x(0, k)=x_{0 k}$ denotes the initial condition of the system at the $k$ th iteration.

For system (1), the ILC law is introduced as follows

$u(t, k)=u(t, k-1)+r(t, k)$,

where $r(t, k)$ is the control updated signal.

In many of the existing ILC approaches, the control updated law is usually chosen as

$$
r(t, k)=f(e(t+1, k), e(t, k), \cdots, e(t-p, k)),
$$

where $e(t, k)=y_{d}(t)-y(t, k)$ is the tracking error and $p$ is a constant. Using this expression, many different types of ILC algorithms can be constructed, such as P-type, PD-type and PID-type. In this paper, we consider the following ILC updated law

$$
r(t, k)=K_{1}(x(t, k)-x(t, k-1))+K_{2} e(t+1, k-1),
$$

where $K_{1}, K_{2}$ are gain metrics to be designed. As shown in, the above updated term contains tracking error signals at current iteration, state signals at current iteration and previous iteration. This scheme can merge the advantages of both feedback control, such as robustness, and ILC, such as extra performance from learning process [24, 32].

As shown in Fig. 1, this paper assumes that the ILC is executed in a network environment. For convenience, we only consider the communication channel existing in the output measurement side. Hence, the data $r(t, k)$ are first quantized by a quantizer, then the quantized data are transmitted to the ILC controller. In this process, the packet loss often occurs. Assume that the controller has an intelligent detection function, it can determine whether the data are lost or not. Then, the ILC law (3) can be described as

$$
u(t, k)=u(t, k-1)+\sigma(t+1, k-1) q(r(t, k)),
$$

where stochastic parameters $\sigma(t, k)$ are the Bernoulli distributed stochastic variables taking the values of 0 and 1 with

$$
\begin{aligned}
& \operatorname{Prob}\{\sigma(t, k)=1\}=E\{\sigma(t, k)\}=\bar{\sigma}, \\
& \operatorname{Prob}\{\sigma(t, k)=0\}=1-E\{\sigma(t, k)\}=1-\bar{\sigma},
\end{aligned}
$$


Figure 1

Structure of the network-based ILC system

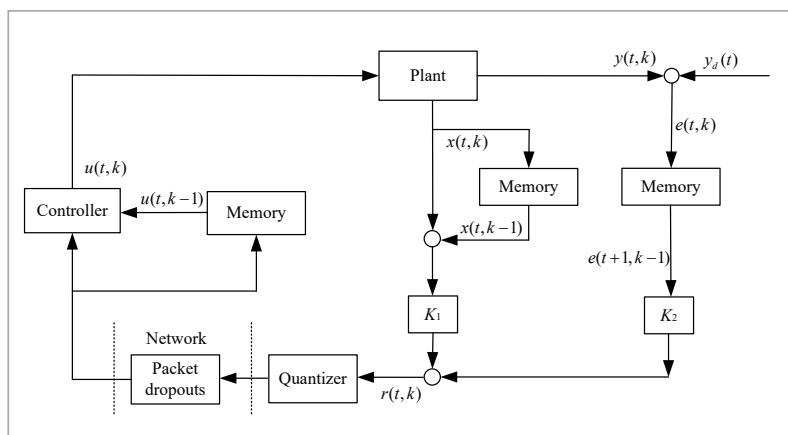

where $\bar{\sigma} \in[0,1]$ is a known constant.

In this paper, it is assumed that the quantizer $q(\cdot)$ is a logarithmic type one and it is defined as

$$
q(\bullet)=\left(q_{1}(\bullet) \quad q_{2}(\bullet) \quad \cdots \quad q_{m}(\cdot)\right)^{T} .
$$

For each $q_{j}(\cdot)(1 \leq j \leq m)$, the set of quantization levels is described by

$$
\begin{aligned}
U=\left\{ \pm z_{i}^{(j)}: z_{i}^{(j)}\right. & \left.=\rho_{j}^{i} z_{0}^{(j)}, i=0, \pm 1, \pm 2, \ldots\right\} \cup\{0\}, \\
0 & <\rho_{j}<1, z_{0}^{(j)}>0 .
\end{aligned}
$$

The logarithmic quantizer $q_{j}(\cdot)$ is defined as

$$
q_{j}(\cdot)=\left\{\begin{array}{ll}
z_{i}^{(j)} & \text { if } \frac{1}{1+\theta_{j}} z_{i}^{(j)}<r_{j} \leq \frac{1}{1-\theta_{j}} z_{i}^{(j)} \\
0 \quad & \text { if } r_{j}=0 \\
-q_{j}\left(-r_{j}\right) & \text { if } r_{j}<0
\end{array}, \quad\right. \text { (7) }
$$

with $\theta_{j}=\frac{1-\rho_{j}}{1+\rho_{j}}$.

To this end, this paper discusses the following problem: For system (1) and a desired trajectory $y_{d}(t)$, design an ILC law (5) under data quantization and random packet dropouts, such that the ILC system is stable and the influence of the iteration-varying disturbances should be as small as possible.

\section{2-D System Representation}

In this section, aiming to analyze the influence of quantization error, the sector bound method in [17] is adopted. That is, for a given signal $r_{j}$, the output of quantizer is $q_{j}\left(r_{j}\right)=\left(1+\mathrm{O}_{j}\left(r_{j}\right)\right) r_{j}$, where $\left|\mathrm{O}_{j}\left(r_{j}\right)\right|<\theta_{j}$. Hence, the effect of quantization error can be described as a sector bound uncertainty as before.

By denoting $\mathrm{O}=\operatorname{diag}\left(\mathrm{O}_{1} \quad \mathrm{O}_{2} \quad \cdots \quad \mathrm{O}_{m}\right)$, from the data quantization in ILC law (5), we have

$$
q(r(t, k))=(I+\mathrm{O}) r(t, k) .
$$

Then the ILC law (4) can be rewritten as

$$
\begin{aligned}
& u(t, k)=u(t, k-1) \\
& +\sigma(t+1, k-1)(I+\mathrm{O}) K\left[\begin{array}{c}
x(t, k)-x(t, k-1) \\
e(t+1, k-1)
\end{array}\right],
\end{aligned}
$$

where $K=\left[\begin{array}{ll}K_{1} & K_{2}\end{array}\right]$.

Defining

$$
\begin{aligned}
& \eta(t, k)=x(t-1, k+1)-x(t-1, k), \\
& \delta u(t, k)=u(t, k)-u(t, k-1) .
\end{aligned}
$$

From and, we can obtain

$$
\begin{aligned}
& \eta(t+1, k) \\
= & (A+\Delta A) \eta(t, k)+(B+\Delta B) \delta u(t-1, k+1) \\
& +B_{1} \tilde{w}(t, k),
\end{aligned}
$$

and

$$
\begin{aligned}
& e(t, k+1)-e(t, k) \\
& =-C(A+\Delta A) \eta(t, k) \\
& -C(B+\Delta B) \delta u(t-1, k+1)-C B_{1} \tilde{w}(t, k) .
\end{aligned}
$$

Substituting (8) into (9) and (10), we can obtain that

$$
\left[\begin{array}{l}
\eta(t+1, k) \\
e(t, k+1)
\end{array}\right]=(\tilde{A}+\sigma(t, k) \tilde{B} \tilde{K})\left[\begin{array}{l}
\eta(t, k) \\
e(t, k)
\end{array}\right]+\bar{B}_{1} \tilde{w}(t, k),
$$

where

$$
\tilde{A}=\bar{A}+\Delta \bar{A}, \tilde{B}=\bar{B}+\Delta \bar{B}, \tilde{K}=(I+\mathrm{O}) K
$$

and

$$
\begin{aligned}
& \bar{A}=\left[\begin{array}{cc}
A & 0 \\
-C A & I
\end{array}\right], \bar{B}=\left[\begin{array}{c}
B \\
-C B
\end{array}\right], \bar{B}_{1}=\left[\begin{array}{c}
B_{1} \\
-C B_{1}
\end{array}\right] . \\
& \Delta \bar{A}=\left[\begin{array}{cc}
\Delta A & 0 \\
-C \Delta A & 0
\end{array}\right], \Delta \bar{B}=\left[\begin{array}{c}
\Delta B \\
-C \Delta B
\end{array}\right],
\end{aligned}
$$




$$
\begin{aligned}
& \Delta \bar{A}=\bar{E} \Sigma \bar{F}_{1}, \Delta \bar{B}=\bar{E} \Sigma \bar{F}_{2}, \bar{E}=\left[\begin{array}{c}
E \\
-C E
\end{array}\right], \\
& \bar{F}_{1}=\left[\begin{array}{ll}
F_{1} & 0
\end{array}\right], \bar{F}_{2}=F_{2} .
\end{aligned}
$$

Denoting

$$
\begin{aligned}
& \eta(t, k)=X^{h}(t, k), \\
& e(t, k)=X^{v}(t, k), \\
& \omega(t, k)=\tilde{w}(t, k),
\end{aligned}
$$

we have

$$
\begin{aligned}
{\left[\begin{array}{l}
X^{h}(t+1, k) \\
X^{v}(t, k+1)
\end{array}\right] } & =(\tilde{A}+\sigma(t, k) \tilde{B} \tilde{K})\left[\begin{array}{l}
X^{h}(t, k) \\
X^{v}(t, k)
\end{array}\right] \\
& +\bar{B}_{1} \omega(t, k) .
\end{aligned}
$$

Notice that the closed-loop system is described as a 2-D Roesser system in (11). The objective of this paper is to find feasible matrices $K_{1}, K_{2}$ such that the 2-D system (11) is stable and has an $H_{\infty}$ disturbance attenuation level. It is worth pointing out that the existence of random variable $\sigma(t, k)$ leads to that the 2-D system (11) is a stochastic system. Hence, the stability definition under the formwork of stochastic system needs to be introduced before proceeding further discussion.

Definition 1 ([18]): Consider the 2-D system. If $\lim _{t+k \rightarrow \infty} E\left\{\|x(t, k)\|^{2}\right\}=0$ holds for every bounded initial condition and $\omega(t, k)=0$, where $x(t, k)=\left[X^{h}(t, k), X^{v}(t, k)\right]^{T}$, then the system is said to be mean-square asymptotically stable.

Definition 2 ( $H_{\infty}$ performance): Given a scalar $\gamma>0$, if the 2-D system satisfies Definition 1 , in addition, $\|x\|_{E}<\gamma\|\omega\|_{2}$ holds for any external disturbance $\omega \in[0, \infty)$, where

$$
\|x\|_{E}=\sqrt{E\left\{\sum_{t=0}^{\infty} \sum_{k=0}^{\infty}\|x(t, k)\|^{2}\right\}},\|\omega\|_{2}=\sqrt{\sum_{t=0}^{\infty} \sum_{k=0}^{\infty}\|\omega(t, k)\|^{2}},
$$

then the 2-D system (11) is mean-square and has an $H_{\infty}$ disturbance attenuation level.

Based on the above two definitions, the objective of this paper is to design an ILC law in form of (5), such that the 2-D stochastic system is mean-square and asymptotically stable and has an $H_{\infty}$ disturbance attenuation level.

\section{Ho ILC Design}

In this section, we first give a sufficient condition to satisfy Definition 2 for system by using a two-dimensional Lyapunov function. Then a feasible controller can be obtained based on the condition of solving the linear matrix inequality.

Define $\tilde{\sigma}(t, k)=\sigma(t, k)-\bar{\sigma}$. It is obvious that

$$
E\{\tilde{\sigma}(t, k)\}=0, E\{\tilde{\sigma}(t, k) \tilde{\sigma}(t, k)\}=\bar{\sigma}(1-\bar{\sigma})
$$

Then the 2-D system (11) can be rewritten as

$$
\begin{aligned}
{\left[\begin{array}{l}
x^{h}(t+1, k) \\
x^{v}(t, k+1)
\end{array}\right] } & =(\tilde{A}+\bar{\sigma} \tilde{B} \tilde{K}+\tilde{\sigma}(t, k) \tilde{B} \tilde{K})\left[\begin{array}{l}
x^{h}(t, k) \\
x^{v}(t, k)
\end{array}\right] \\
& +\bar{B}_{1} \omega(t, k) .
\end{aligned}
$$

Theorem 1: Assume that the packet missing rates $\bar{\sigma}$ and the matrices $\tilde{A}, \tilde{B}, \bar{B}_{1}, \tilde{K}$ are all known. Given a scalar $\gamma>0$, if there exist positive definite matrices $P_{1}, P_{2}$ satisfying

$$
\left[\begin{array}{cc}
\Psi+I & * \\
\bar{B}_{1}^{T} P(\tilde{A}+\bar{\sigma} \tilde{B} \tilde{K}) & \bar{B}_{1}^{T} P \bar{B}-\gamma^{2} I
\end{array}\right]<0,
$$

where

$$
\begin{aligned}
\Psi & =(\tilde{A}+\bar{\sigma} \tilde{B} \tilde{K})^{T} P(\tilde{A}+\bar{\sigma} \tilde{B} \tilde{K}), \\
& +\phi^{2}(\tilde{B} \tilde{K})^{T} P(\tilde{B} \tilde{K})-P, \\
P & \triangleq \operatorname{diag}\left\{P_{1}, P_{2}\right\}>0, \phi^{2}=\bar{\sigma}(1-\bar{\sigma}),
\end{aligned}
$$

then the 2-D system is mean-square asymptotically stable and has an $H_{\infty}$ performance.

Proof. We first consider the stability of the 2-D system without disturbance. In this case, the system (12) becomes

$$
\left[\begin{array}{l}
x^{h}(t+1, k) \\
x^{v}(t, k+1)
\end{array}\right]=(\tilde{A}+\bar{\sigma} \tilde{B} \tilde{K}+\tilde{\sigma}(t, k) \tilde{B} \tilde{K})\left[\begin{array}{l}
x^{h}(t, k) \\
x^{v}(t, k)
\end{array}\right]
$$

and the condition (13) implies

$(\tilde{A}+\bar{\sigma} \tilde{B} \tilde{K})^{T} P(\tilde{A}+\bar{\sigma} \tilde{B} \tilde{K})+\phi^{2}(\tilde{B} \tilde{K})^{T} P(\tilde{B} \tilde{K})$

$-P<0$. 
According to Theorem 1 in [11], we can obtain that the system (14) is mean-square asymptotically stable.

Now, we prove that the 2-D stochastic system (12) has the $H_{\infty}$ disturbance attenuation level.

Assume that the initial boundary conditions of the system are zero, that is, $x^{h}(0, i)=0, x^{v}(i, 0)=0$ for all $i$. Firstly, we define the following index

$$
\begin{aligned}
J & \triangleq E\left\{\left[\begin{array}{l}
x^{h}(t+1, k) \\
x^{v}(t, k+1)
\end{array}\right]^{T} P\left[\begin{array}{l}
x^{h}(t+1, k) \\
x^{v}(t, k+1)
\end{array}\right] \mid \tilde{x}\right\}-\tilde{x}^{T} P \tilde{x}, \\
& =\tilde{x}^{T} \Psi \tilde{x}+\tilde{x}^{T}(\tilde{A}+\bar{\sigma} \tilde{B} \tilde{K})^{T} P \bar{B}_{1} \omega \\
& +\omega^{T} \bar{B}_{1}^{T} P(\tilde{A}+\bar{\sigma} \tilde{B} \tilde{K}) \tilde{x}+\omega^{T} \bar{B}_{1}^{T} P \bar{B}_{1} \omega .
\end{aligned}
$$

Another index is introduced as

$$
\Pi \triangleq \tilde{x}^{T} \tilde{x}-\gamma^{2} \omega^{T} \omega+J=\xi^{T} \Omega \xi,
$$

where

$$
\begin{aligned}
& \xi=\left[\begin{array}{ll}
\tilde{x}^{T} & \omega^{T}
\end{array}\right]^{T}, \\
& \Omega=\left[\begin{array}{cc}
\Psi+I & * \\
\bar{B}_{1}^{T} P(\tilde{A}+\bar{\sigma} \tilde{B} \tilde{K}) & \bar{B}_{1}^{T} P \bar{B}_{1}-\gamma^{2} I
\end{array}\right] .
\end{aligned}
$$

From the condition (13), for any $\xi \neq 0$, we have $\Pi<0$. Taking the expectation of both sides yields

$$
\begin{aligned}
& E\left\{\left[\begin{array}{l}
x^{h}(t+1, k) \\
x^{v}(t, k+1)
\end{array}\right]^{T} P\left[\begin{array}{l}
x^{h}(t+1, k) \\
x^{v}(t, k+1)
\end{array}\right] \mid \tilde{x}\right\} \\
& <E\left\{\tilde{x}^{T} P \tilde{x}-\tilde{x}^{T} \tilde{x}\right\}+\gamma^{2} \tilde{w}^{T} \tilde{w} .
\end{aligned}
$$

Due to the relationship (17), similar to the proof process of Theorem 1 in [11], we can obtain

$$
\begin{aligned}
& E\left\{\sum_{i=0}^{\infty} \sum_{j=0}^{i} \tilde{x}(i-j, j)^{T} \tilde{x}(i-j, j)\right\} \\
& \quad<\gamma^{2} \sum_{i=0}^{\infty} \sum_{j=0}^{i} \tilde{w}(i-j, j)^{T} \tilde{w}(i-j, j) \\
& \quad+\sum_{i=0}^{\infty}\left(E\left\{x^{v}(i, 0)^{T} P_{2} x^{v}(i, 0)\right\}+E\left\{x^{h}(0, i)^{T} P_{1} x^{h}(0, i)^{T}\right\}\right) .
\end{aligned}
$$

Hence,

$\|\tilde{x}\|_{E}<\gamma^{2}\|\tilde{w}\|_{2}$.
This completes the proof.

Remark 1: In Theorem 1, we give a sufficient condition for 2-D systems with communication constraints, including data dropouts and data quantization. This result is still applicable to the 2-D system without communication constraints. If there is no communication constraint between the controller and plant, then $\mathrm{O}=\mathbf{0}, \bar{\sigma}=1$ and $\phi=0$. In this case, the considered 2-D system is a deterministic one and the mean-square asymptotically stable condition becomes

$$
\left[\begin{array}{cc}
(\tilde{A}+\tilde{B} K)^{T} P(\tilde{A}+\tilde{B} K)-P+I & * \\
\bar{B}_{1}^{T} P(\tilde{A}+\tilde{B} K) & \bar{B}_{1}^{T} P \bar{B}_{1}-\gamma^{2} I
\end{array}\right]<0,
$$

which is also obtained by the results in $[15,32,34]$.

Theorem 1 only gives a sufficient condition based on an assumption that the system matrices $\tilde{A}, \tilde{B}, \bar{B}, \tilde{K}$ and parameters $\bar{\sigma}$ are all known. However, the aim of this paper is to design the ILC gain matrices $K_{1}$ and $K_{2}$. In the following, we will give the ILC design method based on the condition in Theorem 1 .

Lemma 1 [5]: Assume $X, Y$ are matrices or vectors with appropriate dimensions. For any scalar $\varepsilon>0$ and matrix $\Delta$ with appropriate dimensions satisfying $\Delta \Delta^{T} \leq I$, the following inequality holds

$$
X \Delta Y+Y^{T} \Delta^{T} X^{T} \leq \varepsilon X X^{T}+\varepsilon^{-1} Y^{T} Y .
$$

Now, we can give the following result.

Theorem 2: If there exist a positive definite matrix $Q$, a matrix $M$ and scalar $\varepsilon>0$ such that the following LMI holds

$$
\left[\begin{array}{cccccccc}
-Q & & & & & & & \\
0 & -\gamma^{2} I & & & & * & & \\
\bar{A} Q+\bar{\sigma} \bar{B} M & \bar{B}_{1} & \Omega_{1} & & & & & \\
\phi \bar{B} M & 0 & \Omega_{2} & \Omega_{3} & & & & \\
Q & 0 & 0 & 0 & -I & & & \\
\overline{F_{1}} Q & 0 & 0 & 0 & 0 & -\varepsilon I & & \\
\sqrt{2} \theta M & 0 & 0 & 0 & 0 & 0 & -\varepsilon I & \\
\bar{F}_{2} M & 0 & 0 & 0 & 0 & 0 & 0 & -\varepsilon I
\end{array}\right]<0,(19)
$$

where

$$
\begin{gathered}
\Omega_{1}=-Q+\varepsilon \bar{E} \bar{E}^{T}+\varepsilon \bar{\sigma} \bar{B}(\bar{\sigma} \bar{B})^{T} \\
+\varepsilon\left(1+\beta^{2}\right) \bar{\sigma} \bar{E}(\bar{\sigma} \bar{E})^{T},
\end{gathered}
$$




$$
\begin{aligned}
\Omega_{2} & =\varepsilon \phi \bar{B}(\bar{\sigma} \bar{B})^{T}+\varepsilon\left(1+\beta^{2}\right) \phi \bar{E}(\bar{\sigma} \bar{E})^{T}, \\
\Omega_{3} & =-Q+\varepsilon \phi \bar{B}(\phi \bar{B})^{T}+\varepsilon\left(1+\beta^{2}\right) \phi \bar{E}(\phi \bar{E})^{T}, \\
\beta & =\sqrt{\max \left(a b s\left(\lambda\left(\bar{F}_{2} \bar{F}_{2}^{T}\right)\right)\right)},
\end{aligned}
$$

then the 2-D system is mean-square asymptotically stable and has an $H_{\infty}$ performance. Furthermore, when the above condition is satisfied, a feasible controller gain can be given as below

$$
K=M Q^{-1}
$$

Proof. The LMI can be rewritten as

$$
\begin{aligned}
& {\left[\begin{array}{ccc}
(\tilde{A}+\bar{\sigma} \tilde{B} \tilde{K})^{T} & \phi(\tilde{B} \tilde{K})^{T} & I \\
\bar{B}_{1}{ }^{T} & 0 & 0
\end{array}\right]\left[\begin{array}{ccc}
P & & * \\
0 & P & \\
0 & 0 & I
\end{array}\right]} \\
& {\left[\begin{array}{cc}
(\tilde{A}+\bar{\sigma} \tilde{B} \tilde{K}) & \bar{B}_{1} \\
\phi(\tilde{B} \tilde{K}) & 0 \\
I & 0
\end{array}\right]+\left[\begin{array}{cc}
-P & 0 \\
0 & -\gamma^{2} I
\end{array}\right]<0 .}
\end{aligned}
$$

By using Schur Complement, (24) can be transformed into the following LMI

$$
\left[\begin{array}{ccccc}
-P & & & & \\
0 & -\gamma^{2} I & & * & \\
\tilde{A}+\bar{\sigma} \tilde{B} \tilde{K} & \bar{B}_{1} & -P^{-1} & & \\
\phi \tilde{B} \tilde{K} & 0 & 0 & -P^{-1} & \\
I & 0 & 0 & 0 & -I
\end{array}\right]<0 .
$$

In addition, by using Lemma 1 , it can be transformed into

$$
\begin{aligned}
& {\left[\begin{array}{ccccc}
-P & & & \\
0 & -\gamma^{2} I & & * & \\
\bar{A}+\bar{\sigma} \bar{B} K & \bar{B}_{1} & \Xi_{1} & & \\
\phi \bar{B} K & 0 & \Xi_{2} & \Xi_{3} & \\
I & 0 & 0 & 0 & -I
\end{array}\right]+} \\
& \varepsilon^{-1}\left[\begin{array}{ccc}
\bar{F}_{1}^{T} & \sqrt{2} \theta K^{T} & \left(\bar{F}_{2} K\right)^{T} \\
0 & 0 & 0 \\
0 & 0 & 0 \\
0 & 0 & 0 \\
0 & 0 & 0
\end{array}\right]\left[\begin{array}{ccccc}
\bar{F}_{1} & 0 & 0 & 0 & 0 \\
\sqrt{2} \theta K & 0 & 0 & 0 & 0 \\
\bar{F}_{2} K & 0 & 0 & 0 & 0
\end{array}\right]<0
\end{aligned}
$$

where

$$
\begin{aligned}
& \Xi_{1}=-P^{-1}+\varepsilon \bar{E} \bar{E}^{T}+\varepsilon \bar{\sigma} \bar{B}(\bar{\sigma} \bar{B})^{T}+\varepsilon\left(1+\beta^{2}\right) \bar{\sigma} \bar{E}(\bar{\sigma} \bar{E})^{T}, \\
& \Xi_{2}=\varepsilon \phi \bar{B}(\bar{\sigma} \bar{B})^{T}+\varepsilon\left(1+\beta^{2}\right) \phi \bar{E}(\bar{\sigma} \bar{E})^{T}, \\
& \Xi_{3}=-P^{-1}+\varepsilon \phi \bar{B}(\phi \bar{B})^{T}+\varepsilon\left(1+\beta^{2}\right) \phi \bar{E}(\phi \bar{E})^{T} .
\end{aligned}
$$

Define $Q=P^{-1}$. Pro- and post multiplying $\operatorname{diag}\{Q, I, I, I, I, I, I, I\}$ for (25) and setting $M=K Q$, the proof can be obtained.

Remark 2: Theorem 2 gives another LMI condition for the 2-D stochastic system. If this condition is satisfied, the mean-square asymptotic stability can be guaranteed and, meanwhile, the system has a $H_{\infty}$ disturbance attenuation level. Furthermore, this condition can also supply a feasible solution of ILC gain by solving LMI.

Remark 3: For a fixed $\gamma$, the feasible solution of LMI (22) can give a sub-optimal $H_{\infty}$ ILC design result. If $\gamma$ is not fixed, the optimal performance index $\gamma$ can be searched by solving the following convex programming problem:

$$
\min _{Q_{1}, Q_{2}, M_{1}, M_{2}, \varepsilon} \gamma^{2}
$$

s.t. (22).

In this case, we can give an optimal $H_{\infty}$ ILC design result.

\section{An Illustrative Example}

Inthissection, theproposed designisused for aninjection molding process. Injection molding process has the characteristic of repeated operation, where some important process variables are controlled to track certain desired trajectories repetitively to ensure the product quality [32]. In injection molding process, injection velocity is an important variable in filling stage. It is controlled by operating the opening of a hydraulic valve. The injection molding velocity control systems can be described as the following state-space model [32]:

$$
\left\{\begin{aligned}
x(k, t+1) & =\left(\left[\begin{array}{cc}
1.58 & -0.59 \\
1 & 0
\end{array}\right]+\Delta A\right) x(k, t) \\
& +\left(\left[\begin{array}{l}
1 \\
0
\end{array}\right]+\Delta B\right) u(k, t)+\left[\begin{array}{l}
1 \\
1
\end{array}\right] w(k, t), \\
y(k, t) & =\left[\begin{array}{ll}
1.69 & -1.42] x(k, t),
\end{array}\right.
\end{aligned}\right.
$$


where $\Delta A, \Delta B$ may be caused by model mismatch or nonlinearity of the injection modeling process. In this simulation, it is assumed that $\Delta A, \Delta B$ only varied on iteration domain randomly. That is

$$
\begin{aligned}
& E=\left[\begin{array}{cc}
0.5 & 0.5 \\
0 & 0
\end{array}\right], F_{1}=\left[\begin{array}{cc}
0.2 & 0 \\
0 & 0.2
\end{array}\right], F_{2}=\left[\begin{array}{l}
0.01 \\
0.01
\end{array}\right], \\
& \Sigma=\left[\begin{array}{cc}
\xi_{1}(k) & \\
\xi_{2}(k)
\end{array}\right],\left|\xi_{1}(k)\right|<1,\left|\xi_{2}(k)\right|<1,
\end{aligned}
$$

where $\xi_{1,2}(k)$ are unknown variables. $w(k, t)$ is a random disturbance and it is selected as a uniformly distributed sequence in $[-0.001,0.001]$.

The desired trajectory is given as:

$$
y_{d}(t)=\left\{\begin{array}{lr}
0.2+0.0025 t, & t \leq 40 \\
0.3, & 40<t \leq 300 \\
0.3+0.0025(t-300), & 300<t \leq 380 \\
0.5, & 380<t \leq 800
\end{array} .\right.
$$

For the initial conditions, it is assumed that $x_{1}(0, k)=x_{2}(0, k)=0$ for all $k$. The control input is given as $u(t, 0)=0$ for all $t$. The parameters of the logarithmic quantizer are $\rho=0.8, z_{0}=0.1$. In this simulation, we consider two different packet dropouts, that is

Case 1: $\bar{\sigma}=1$,

Case 2: $\bar{\sigma}=0.8$.

Obviously, in the first case, there is no data loss, and in the second case, there is a data loss of $20 \%$. Based on the Theorem 2, the corresponding controllers can be computed as $\gamma_{\text {opt }}=10.5$, $K_{1}=\left[\begin{array}{ll}-0.80 & 0.59\end{array}\right], K_{2}=0.52$ for Case 1 and $\gamma_{\text {opt }}=12.8, K_{1}=\left[\begin{array}{ll}-0.92 & 0.63\end{array}\right], K_{2}=0.37$ for Case 2. For Case 1, system outputs at 5th, 10th and 50th iterations are plotted in Fig. 2. It can be seen that the tracking error can be reduced to within 0.01 at the 5 th iteration. As the number of iterations increases, the tracking error becomes smaller and smaller. After the 50th iteration, a satisfied tracking performance can be obtained. Notice that the small oscillation at the 50th iteration is produced by the effect of random disturbance. Furthermore, the tracking error is also plotted in Fig. 3 to show the convergence procedure clearly, where the horizontal coordinate is the number of iterations and the vertical coordinate is maximum tracking error.
Figure 2

System output profiles for Case 1: (a) 5 th iteration. (b) 10th iteration. (c) 50th iteration

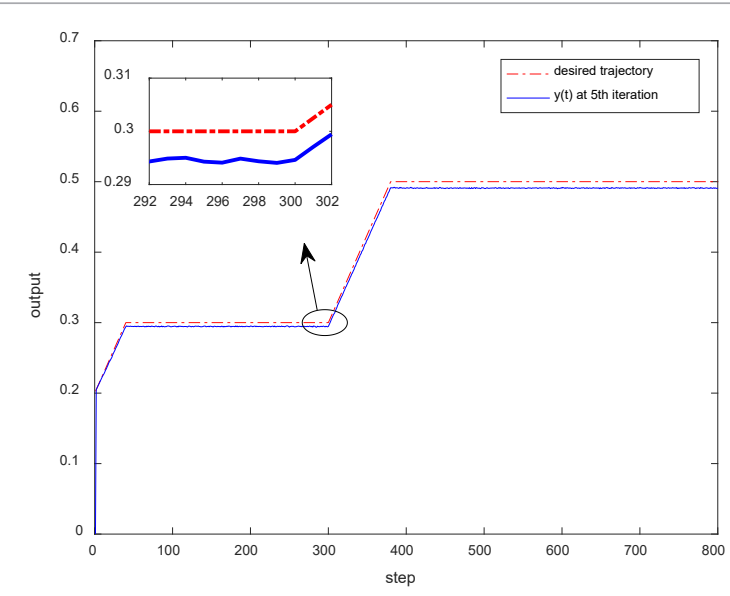

a

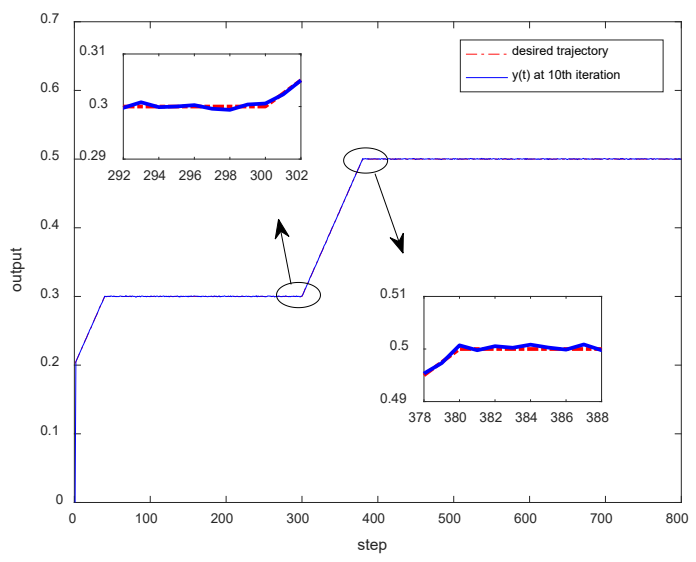

b

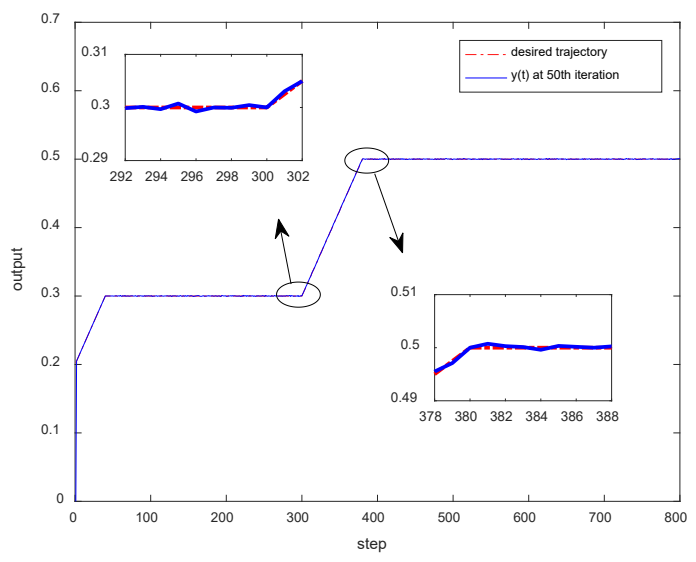

c 
Figure 3

Max tracking error on iteration domain for Case 1

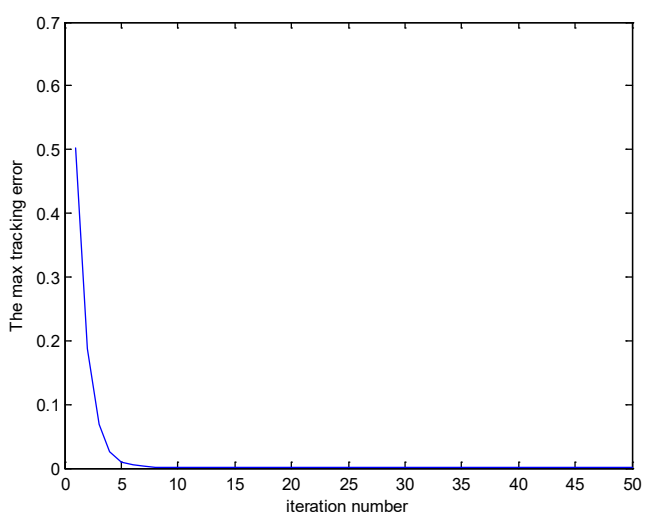

For Case 2, the system outputs in three different iterations are shown in Fig. 4 and the max tracking errors in the iteration learning process are given in Fig. 5. It can be seen that the tracking error only can be reduced to within 0.1 at the 5th iteration. Thus the tracking error reduced to within 0.01 at the 10th iteration. This implies that the convergence speed of Case 2 is slower than Case 1. Hence, the convergence speed of tracking error gets slower as dropout rate increases. We can also observe that a satisfied tracking performance is obtained after the 50th iteration in Fig. 5, which implies

\section{Figure 4}

System output profiles for Case 2: (a) 5th iteration. (b) 10th iteration. (c) 50th iteration

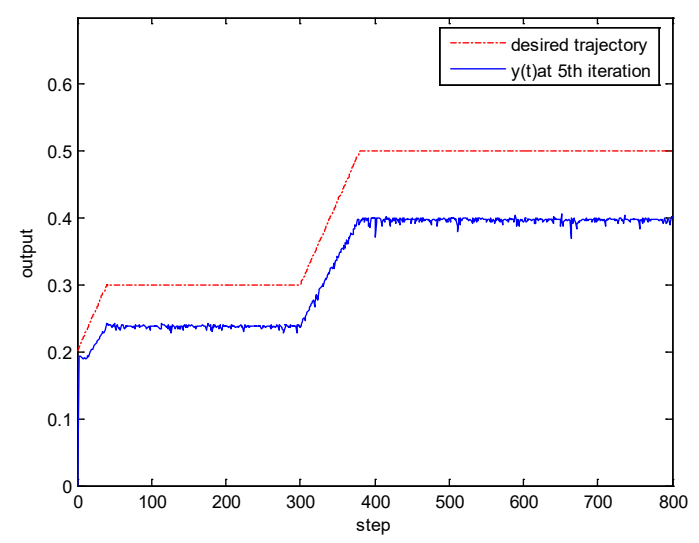

a

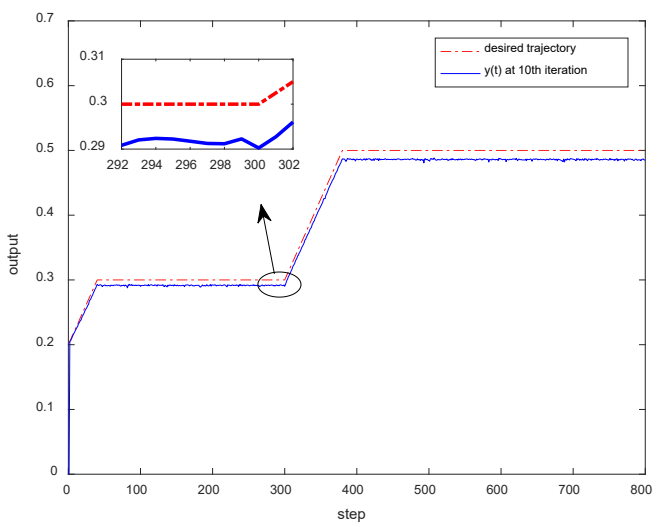

b

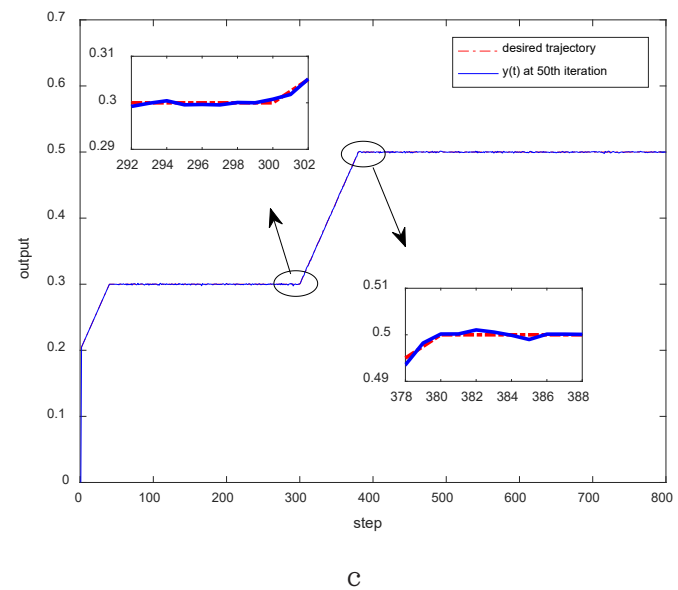

Figure 5

Max tracking error on iteration domain for Case 2

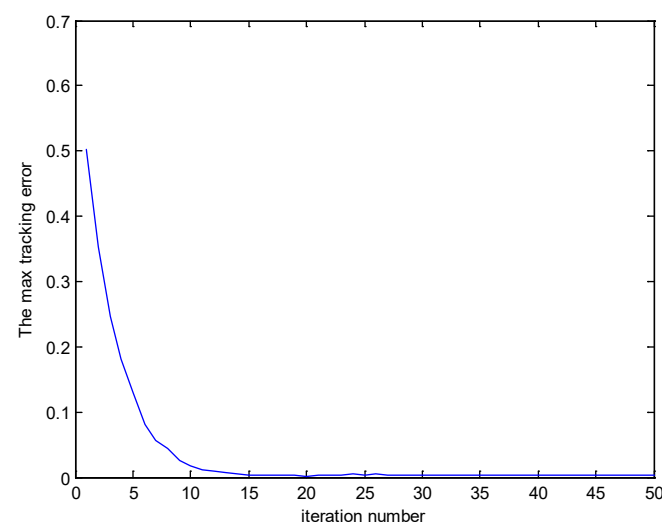


that even though there exists a large tracking error at the start iteration due to the effect of packet dropouts and data quantization, better tracking performance can also be obtained through iteration learning process.

Notice that the oscillation in Fig. 4 (c) is greater than that in Case 1 in Fig. 2 (c), which also illustrates that the data dropout will enlarge the effect of random disturbance for the ILC systems.

\section{Conclusions}

In this paper, a robust $\mathrm{H} \infty$ ILC has been designed for network-based uncertain systems with both random data dropouts and data quantization. It is assumed that the system state and tracking error are first quantized through a logarithmic quantizer, which can decode these data into finite quantization

\section{References}

1. Ahn, H. S., Chen, Y., Moore, K. L. Iterative Learning Control: Brief Survey and Categorization. IEEE Transactions on Systems, Man, and Cybernetics-Part C: Applications and Reviews, 2007, 37(6), 1099-1121. https:// doi.org/10.1007/978-1-84628-859-3

2. Ahn, H. S., Chen, Y. Q., Moore, K. L. Discrete-Time Intermittent Iterative Learning Control with Independent Data Dropouts. Proceedings of 17th IFAC World Congress, Korea, 2008, 12442-12447.

3. Alarcon-Aquino, V., Ramirez-Cortes, J. M., Gomez-Gil, P., Starostenko, O., Garcia-Gonzalez, Y. Network Intrusion Detection Using Self-Recurrent Wavelet Neural Network with Multidimensional Radial Wavelons. Information Technology and Control, 2014, 43(4), 347358. https://doi.org/10.5755/j01.itc.43.4.4626

4. Arimoto, S., Kawamura, S., Miyazaki, F. Bettering Operation of Robots by Learning. Journal of Robotic Systems, 1984, 1(2), 123-140. https://doi.org/10.1002/ rob.4620010203

5. Boyd, S., Ghaoui, L. E., Feron, E., Balakrishnan, V. Linear Matrix Inequalities in System and Control Theory. SIAM, Philadelphia, 1994. https://doi. org/10.1137/1.9781611970'777

6. Bristow, D. A., Tharayil, M., Alleyne, A. G. A Survey of Iterative Learning Control: A Learning-Based Method for High-Performance Tracking Control. IEEE Control levels. Furthermore, the model of packet dropout is described as the Bernoulli binary value sequences with known probability. Then the considered ILC systems can be transformed into a 2-D stochastic system. An asymptotical stability condition has been developed for such 2-D system and then a feasible ILC design approach is given. It is shown that the proposed ILC design can suppress the influence of random data dropouts, data quantization and the iteration-varying disturbances effectively.

\section{Acknowledgement}

This work is supported by the National Natural Science Foundation of China (Nos. 61573129), the Innovative Scientists and Technicians Team of Henan Polytechnic University (T2017-1) and the Program for Science and Technology Innovation Talents in Universities of Henan Province (16HASTIT046).

Systems, 2006, 26(3), 96-114. https://doi.org/10.1109/ MCS.2006.1636313

7. Brockett, R. W., Liberzon, D. Quantized Feedback Stabilization of Linear Systems. IEEE Transactions on Automatic Control, 2000, 45(7), 1279-1289. https://doi. org/10.1109/9.867021

8. Bu, X. H., Yu, F. S., Hou, Z. S., Wang, F. Z. Iterative Learning Control for a Class of Nonlinear Systems with Random Packet Losses. Nonlinear Analysis: Real World Applications, 2013, 14(1), 567-580. https://doi. org/10.1016/j.nonrwa.2012.07.017

9. Bu, X. H., Hou, Z. S., Yu, F. S., Wang, F. Z. Hळ Iterative Learning Controller Design for a Class of Discrete-Time Systems with Data Dropouts. International Journal of Systems Science, 2014, 45(9), 1902-1912. https://doi. org/10.1080/00207721.2012.757815

10. Bu, X. H., Wang, T. H., Hou, Z. S., Chi, R. H. Iterative Learning Control for Discrete-time Systems with $\mathrm{Qu}$ antised Measurements. IET Control Theory \& Applications. 2015, 9(9), 1455-1460. https://doi.org/10.1049/ iet-cta.2014.1056

11. Bu, X. H., Hou, Z. S., Jin, S. T. An Iterative learning Control Design Approach for Networked Control Systems with Data Dropouts. International Journal of Robust and Nonlinear Control, 2016, 26(1), 91-109. https://doi. org/10.1002/rnc.3300 
12. Chi,R.H.,Hou,Z.S., Xu, J.X. AdaptiveILCfor a Class ofDiscrete-Time Systems with Iteration-Varying Trajectory and Random Initial Condition. Automatica, 2008, 44 (8), 22072213. https://doi.org/10.1016/j.automatica.2007.12.004

13. Chi, R. H., Wang, D. W., Hou, Z. S., Jin, S. T. Data-Driven Optimal Terminal Iterative Learning Control. Journal of Process Control, 2012, 22 (10), 2026-2037. https:// doi.org/10.1016/j.jprocont.2012.08.001

14. Dong, S., Wang, Y. Q. ILC for Networked Nonlinear Systems with Unknown Control Direction Through Random Lossy Channel. Systems \& Control Letters, 2015, 7\%, 30-39. https://doi.org/10.1016/j.sysconle.2014.12.008

15. Du, C., Xie L., Zhang C. Hळ Control and Robust Stabilization of Two-Dimensional Systems in Roesser Models. Automatica, 2011, 37(2), 205-211. https://doi. org/10.1016/S0005-1098(00)00155-2

16. Faraji-Niri, M., Jahed-Motlagh, M. Stochastic Stability and Stabilization of Semi-Markov Jump Linear Systems with Uncertain Transition Rates. Information Technology and Control, 2017, 46(1), 37-52. https://doi. org/10.5755/j01.itc.46.1.13881

17. Fu, M., Xie, L. The Sector Bound Approach to Quantized Feedback Control. IEEE Transactions on Automatic Control, 2005, 50(11), 1698-1710. https://doi. org/10.1109/TAC.2005.858689

18. Gao, H., Lam, J., Xu, S., Wang, C. Stability and Stabilization of Uncertain 2-D Discrete Systems with Stochastic Perturbation. Multidimensional Systems and Signal Processing, 2005, 16(1), 85-106. https://doi. org/10.1007/s11045-004-4739-y

19. Gupta, R. A., Chow, M. Y. Networked Control System: Overview and Research Trends. IEEE Transactions on Industrial Electronics, 2010, 57 (7), 2527-2535. https:// doi.org/10.1109/TIE.2009.2035462

20. Hespanha, J. P., Naghshtabrizi, P., Xu, Y. G. A Survey of Recent Results in Networked Control Systems, Proceedings of the IEEE, 2007, 95(1), 138-162. https://doi. org/10.1109/JPROC.2006.887288

21. Hladowski, L., Galkowski, K., Cai, Z., Rogers, E., Freeman, C. T., Lewin, P. L. Experimentally Supported 2-D Systems Based Iterative Learning Control Law Design for Error Convergence and Performance. Control Engineering Practice, 2010, 18(4), 339-348. https://doi. org/10.1016/j.conengprac.2009.12.003

22. Hou, Z. S., Xu, J. X., Yan, J. W. An Iterative Learning Approach for Density Control of Freeway Traffic Flow via Ramp Metering. Transportation Research Part C, 2008, 16 (1), 71-97. https://doi.org/10.1016/j.trc.2007.06.007

23. Hou, Z. S., Wang, Z. From Model-Based Control to Data-Driven Control: Survey, Classification and Perspective. Information Sciences, 2013, 235, 3-35. https://doi. org/10.1016/j.ins.2012.07.014
24. Li, X. D., Chow, T. W. S., Ho, J. K. L. 2-D System Theory Based Iterative Learning Control for Linear Continuous Systems with Time Delays. IEEE Transactions on Circuits and Systems I: Regular Papers, 2005, 52(7), 1421-1430. https://doi.org/10.1109/TCSI.2005.851706

25. Liberzon, D. Hybrid Feedback Stabilization of Systems with Quantized Signals. Automatica, 2003, 39(9), 15431554. https://doi.org/10.1016/S0005-1098(03)00151-1

26. Liu, C. P., Xu, J. X., Wu, J. Iterative Learning Control for Network Systems with Communication Delay or Data Dropout. Proceedings of 48rd IEEE Conference on Decision and Control, China, 2009, 4858-4863. https://doi. org/10.1109/CDC.2009.5400749

27. Liu, Q., Yang, G. H. Quantized Feedback Control for Networked Control Systems under Information Limitation. Information Technology and Control, 2011, 40(3), 218-226. https://doi.org/10.5755/j01.itc.40.3.630

28. Ma, X. H., Bu, X. H. A Novel Iterative Learning Control Design for Linear Discrete Time Systems Based on a 2D Roesser System. Information Technology and Control, 2016, 45(4), 384-392.

29. Meng, D., Jia, Y., Du, J., Yu, F. Data-Driven Control for Relative Degree Systems via Iterative Learning. IEEE Transactions on Neural Network, 2012, 22(12), 22132225. https://doi.org/10.1109/TNN.2011.2174378

30. Peng, C., Tian, Y. C. Networked Hœ Control of Linear Systems with State Quantization. Information Sciences, 2007, 1777(24), 5763-5774. https://doi.org/10.1016/j. ins.2007.05.025

31. Shen, D., Wang, Y. Survey on Stochastic Iterative Learning Control. Journal of Process Control, 2014, 24(12), 64-77. https://doi.org/10.1016/j.jprocont.2014.04.013

32. Shi, J., Gao, F., Wu, T. J. Robust Design of Integrated Feedback and Iterative Learning Control of a Batch Process Based on a 2D Roesser System. Journal of Process Control, 2005, 15(8), 907-924. https://doi.org/10.1016/j. jprocont.2005.02.005

33. Wang, Y., Gao, F., Doyle, F. J. III. Survey on Iterative Learning Control, Repetitive Control, and Run-to-Run Control. Journal of Process Control, 2009, 19(10), 15891600. https://doi.org/10.1016/j.jprocont.2009.09.006

34. Wang, Y., Yang, Y., Zhao, Z. Robust Stability Analysis for an Enhanced ILC-Based PI Controller. Journal of Process Control, 2013, 23(2), 201-214. https://doi.org/10.1016/j.jprocont.2012.08.004

35. Yang, T. C. Networked Control System: A Brief Survey. IEE Proceedings-Control Theory and Applications, 2006, 153(4), 403-412. https://doi.org/10.1049/ip-cta:20050178

36. Zhang, L. X., Gao, H. J., Kaynak, O. Network-Induced Constraints in Networked Control Systems-A Survey. IEEE Transactions on Industrial Informatics, 2013, 9(1), 403-416. https://doi.org/10.1109/TII.2012.2219540 